\title{
T Helper 17/Regulatory $T$ Cell Balance and Experimental Models of Peritoneal Dialysis-Induced Damage
}

\author{
Georgios Liappas, ${ }^{1,2}$ Guadalupe Tirma Gónzalez-Mateo, ${ }^{1,2}$ Pedro Majano, ${ }^{3}$ \\ José Antonio Sánchez- Tomero, ${ }^{4}$ Marta Ruiz-Ortega, ${ }^{5}$ Raquel Rodrigues Díez, ${ }^{5}$ \\ Pilar Martín, ${ }^{6}$ Raquel Sanchez-Díaz, ${ }^{6}$ Rafael Selgas, ${ }^{2}$ \\ Manuel López-Cabrera, ${ }^{1}$ and Abelardo Aguilera Peralta ${ }^{3}$ \\ ${ }^{1}$ Department of Immunology, Centro de Biología Molecular Severo Ochoa (CBM), \\ Consejo Superior de Investigaciones Científicas (CSIC), 28049 Madrid, Spain \\ ${ }^{2}$ Department of Nephrology, Instituto de Investigación del Hospital Universitario La Paz (IdiPAZ), 28046 Madrid, Spain \\ ${ }^{3}$ Department of Molecular Biology, Instituto de La Princesa (IP), 28006 Madrid, Spain \\ ${ }^{4}$ Nephrology Service, Hospital Universitario de La Princesa (IP), 28006 Madrid, Spain \\ ${ }^{5}$ Laboratory of Cellular Biology and Rare Diseases, Fundación Jiménez Díaz, Universidad Autónoma de Madrid (UAM), \\ 28040 Madrid, Spain \\ ${ }^{6}$ Department of Molecular and Vascular Inflammation, Centro Nacional de Investigaciones Cardiovasculares (CNIC), \\ 28029 Madrid, Spain
}

Correspondence should be addressed to Guadalupe Tirma Gónzalez-Mateo; guadalupe.gonzalez@idipaz.es and Abelardo Aguilera Peralta; abelardo.aguilera@salud.madrid.org

Received 18 September 2014; Accepted 29 December 2014

Academic Editor: Monica Fedele

Copyright ( 2015 Georgios Liappas et al. This is an open access article distributed under the Creative Commons Attribution License, which permits unrestricted use, distribution, and reproduction in any medium, provided the original work is properly cited.

\begin{abstract}
Fibrosis is a general complication in many diseases. It is the main complication during peritoneal dialysis (PD) treatment, a therapy for renal failure disease. Local inflammation and mesothelial to mesenchymal transition (MMT) are well known key phenomena in peritoneal damage during PD. New data suggest that, in the peritoneal cavity, inflammatory changes may be regulated at least in part by a delicate balance between $\mathrm{T}$ helper 17 and regulatory $\mathrm{T}$ cells. This paper briefly reviews the implication of the Th17/Tregaxis in fibrotic diseases. Moreover, it compares current evidences described in PD animal experimental models, indicating a loss of Th17/Treg balance (Th17 predominance) leading to peritoneal damage during PD. In addition, considering the new clinical and animal experimental data, new therapeutic strategies to reduce the Th17 response and increase the regulatory $\mathrm{T}$ response are proposed. Thus, future goals should be to develop new clinical biomarkers to reverse this immune misbalance and reduce peritoneal fibrosis in PD.
\end{abstract}

\section{Introduction}

An effective inflammatory response is essential not only for the resolution of infections but also for wound healing after injury. The repair is mediated by the collaboration of various mechanisms launched by an acute inflammatory reaction. This process implicates the release of chemokines and cytokines and the migration of various cells of the immune system. If a sustained inflammatory reaction that is not resolved properly becomes chronic, it could lead to fibrosis due to the accumulation of extracellular matrix (ECM) components [1].

Peritoneal dialysis (PD) is a form of renal replacement therapy alternative to haemodialysis that is widely used around the world for patients suffering from renal failure disease $[2,3]$. The process uses the peritoneum as a semipermeable membrane across which PD fluids (PDFs) and dissolved substances (electrolytes, urea, glucose, and other small molecules) are exchanged from the blood [4]. The peritoneal membrane $(\mathrm{PM})$ acts as a protective barrier against injury 
and pathogens, where humoural and cellular responses are generated. The treatment consists of the instillation and periodical renovation of a hyperosmotic PDF in the peritoneal cavity through a permanent installed catheter. However, the mechanical damage due to PDF instillation and the exposure of peritoneal cells to glucose degradation products (GDPs) and advanced glycation end products (AGEs) (due to the nonphysiological nature of this PDF) generates inflammation. Along these lines, it has been demonstrated in vitro that GDPs and AGEs stimulate $\mathrm{NF} \kappa \mathrm{B}$-mediated transcription and the secretion of cytokines and chemokines by human peritoneal mesothelial cells [5]. In addition, the presence of the catheter [6] and peritonitis episodes during treatment [7] appears to be responsible for various alterations of the PM structure and functionality. The final consequence is the generation of vascular alterations [8] and peritoneal fibrosis $[3,9]$, leading to an ultrafiltration failure $[10,11]$ that impedes the dialysis process (Figure 1). Although in recent decades great effort has been made to improve catheter design [6] and the biocompatible solutions used [12-14], complications are still common $[15,16]$. It has also been demonstrated that inflammation is present in PD patients. Increased serum concentrations of IL-6, TNF- $\alpha$, VEGF, and C-reactive proteins have been reported in patients, suggesting that PD leads to increased systemic inflammation [17]. Moreover, there is evidence in animal models of chronic peritoneal exposure to PDF showing that this procedure induces PM inflammation and fibrosis [18]. The use of an anti-inflammatory drug (a Cox-2 inhibitor) reduced this peritoneal inflammatory response and consequently the fibrosis. This result confirms the role of inflammation in peritoneal fibrosis [19].

Inflammation is driven by various cell populations including macrophages, neutrophils, and lymphocyte subsets. The differentiation of $\mathrm{T}$ cells is crucial for immune and inflammatory responses and its regulation may be a therapeutic target to control peritoneal damage. It has been found that there are different rates between $\mathrm{CD} 4^{+}$and $\mathrm{CD} 8^{+}$ cells in the peritoneum during PD with respect to healthy individuals [20]. It has been postulated that the presence of AGEs is responsible for an increase in the population of $\mathrm{CD}^{+}$(T cytotoxic) lymphocytes [21]. Regarding CD4 ${ }^{+}$(T helper) subsets, in general terms Th1 cells produce high levels of IFN- $\gamma$, while Th2 cells secrete predominantly IL-4 [22]. There is some controversy about the pattern of response that is generated in patients undergoing treatment with PDF. It is described that there is a deviation toward Th2 pattern of PD in stable patients [23]. However, during episodes of acute peritonitis, a Th1 immune response is developed [24, 25]. In addition to these two classical $T$ helper cell subsets (Th1 and Th2), a third and fourth subpopulation, designated regulatory $\mathrm{T}$ (Treg) and T helper 17 (Th17) cells, have emerged as independent differentiation pathways $[26,27]$. While the predominance of Th17 cells induces the secretion of a large number of proinflammatory cytokines, Treg cells restrict inflammatory responses and are associated with immunetolerance [28]. Very little is known about the involvement of these subpopulations in the deterioration of the peritoneum during dialysis. The imbalance between these situations may cause fibroproliferative diseases and could be an important cause of morbidity and mortality.

In this review we discuss the implication of Th17 and Treg cells in kidney function and fibrotic diseases in different animal experimentation models. More specifically we will focus on the origin of peritoneal damage and its relationship with the intraperitoneal presence of these particular subsets of $\mathrm{T}$ lymphocytes, as well as on its clinical implication in peritoneal damage in $\mathrm{PD}$ patients, which frequently results in an inability to continue with the treatment.

\section{T Helper 17 and Regulatory $T$ Cell Differentiation and Their Plasticity}

Th17 cells represent a subset of $\mathrm{T}$ helper cells that secrete mainly interleukin- (IL-) 17 as well as other proinflammatory cytokines, and they have been related to many autoimmune and chronic inflammatory diseases [29]. There is a balance between Th17 and Treg cells that depends on the activation of the transcription factor ROR $\gamma$ t (factors retinoic acid receptor-related orphan receptor $\gamma \mathrm{t}$ ) and Stat3 (signal transducer and activator of transcription 3), or FoxP3 (forkhead box P3) and Stat5, respectively, which regulate the immune response through the secretion of pro- and antiinflammatory cytokines [30-33]. On the other hand, the importance of Treg cells to the maintenance of peripheral tolerance under noninflammatory conditions throughout life has also been confirmed. In fact, mice lacking Treg cells presented a fatal inflammatory response $[34,35]$.

The main cytokines involved in Th17/Treg balance are the TGF- $\beta$ (transforming growth factor beta) and IL-6 (interleukin-6) [27, 36, 37]. IL-6 is strongly induced in cells of the innate immune system upon stimulation of pattern recognition receptors such as toll like receptors (TLR) or C-type receptors. It has been shown that mice lacking IL-6 present a deficiency in the differentiation of effector $\mathrm{T}$ cells $[38,39]$. TGF- $\beta$ in the absence of IL- 6 induces Foxp3, thus pushing T-cell differentiation away from the Th17 transcriptional program and decidedly toward the Treg lineage [33]. Moreover, in the central nervous system, TGF- $\beta$ without the synergy of IL- 6 will force T cells to differentiate through the $\mathrm{T}$ regulatory cell lineage [40]. In contrast, the proinflammatory cytokine IL- 6 in the absence of TGF- $\beta$ activates Stat 3 by phosphorylating it, which overcomes Foxp 3 inhibition of ROR $\gamma \mathrm{t}$ transcriptional activity. This process leads to the upregulation of the IL-23R, thus pushing Tcell differentiation toward a Th17 fate [33]. Therefore the cytokine environment is essential for the predominance of an inflammatory or an anti-inflammatory response (Figure 2).

\section{Th17 and Tissue Fibrosis}

Fibroproliferative diseases such as idiopathic pulmonary, liver, cardiovascular, and renal fibrosis are usually associated with chronic inflammation, as has been described previously [41-45]. When an inflammatory response becomes chronic, the accumulation of ECM is more extensive and the function of the organ is compromised. A number of studies have 


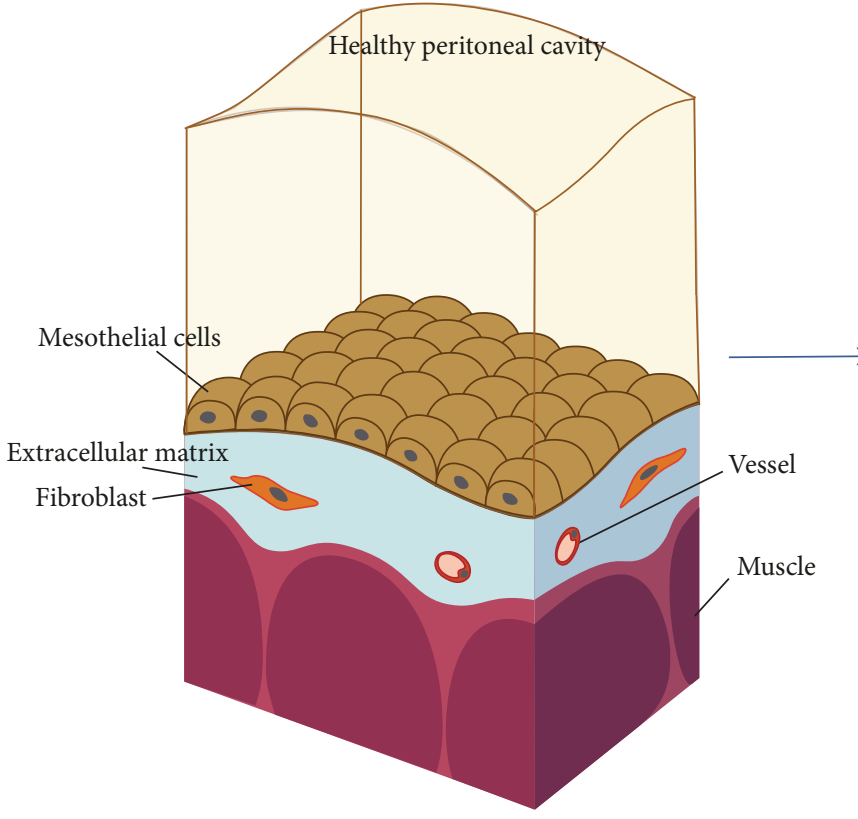

(a)

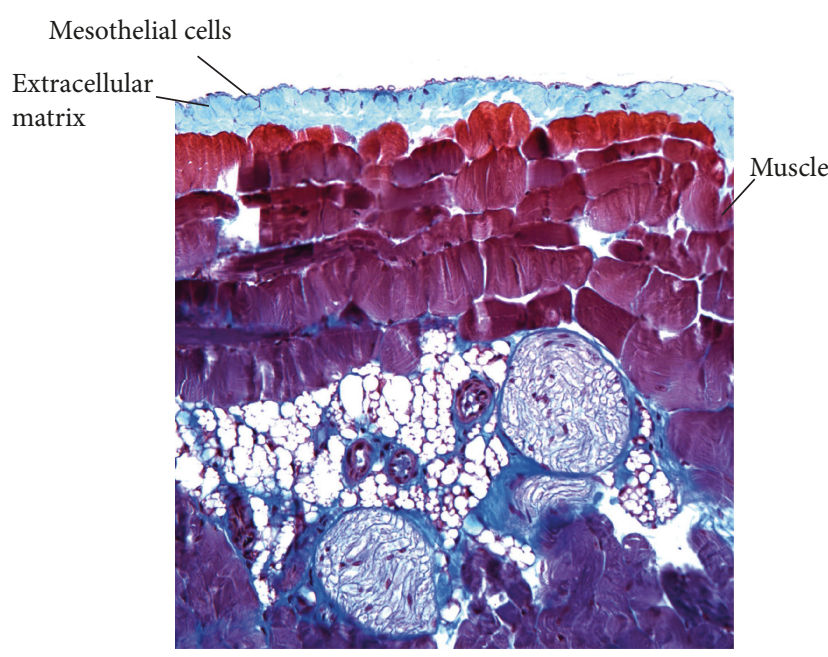

(c)

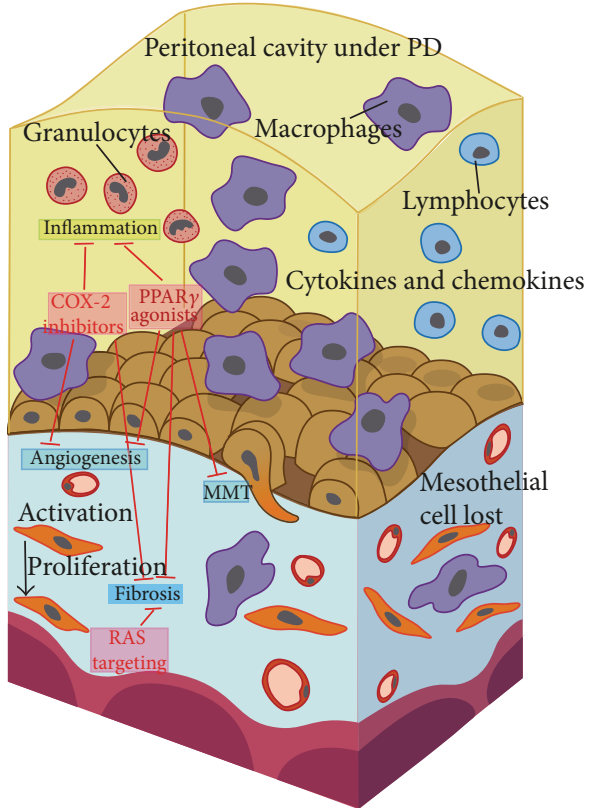

(b)

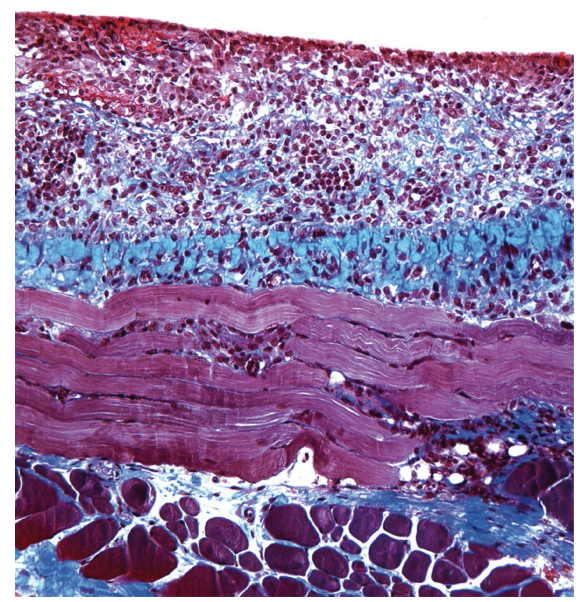

(d)

Figure 1: PM schemas and biopsies representing the normal peritoneal structure and its changes during PD. Possible therapeutic approaches. (a) and (b) are adapted with permission from Aguilera et al. (2013). Available from http://www.intechopen.com/books/the-latestin-peritoneal-dialysis/the-mesothelial-to-mesenchymal-transition-a-pathogenic-and-therapeutic-key-for-peritoneal-membrane- $\mathrm{f}$ [80]. (a) Realistic representation of a healthy peritoneal cavity. A preservation of the mesothelial layer is clearly seen, only a few fibroblasts and vessels are visible, and only a small layer of extracellular matrix lies in the compact zone of the muscle layer. (b) After exposure to PD liquids, the structure of the PM starts to change dramatically with the appearance of more fibroblasts, macrophages, and inflammatory cytokines and finally with deposition of more extracellular matrix (ECM) cells. Inflammation and fibrosis will be the result of these changes. Some drugs like COX-2 inhibitors, PPRA $\gamma$ agonists, or RAS targeting are possible therapeutic strategies to protect from PD complications such as inflammation, angiogenesis, fibrosis, and/or MMT. (c) A peritoneal biopsy of a mouse peritoneal membrane that was treated with physiological saline is shown as control. The PM is well preserved and fibrotic response is absent. (d) Peritoneal biopsy of a mouse PM that was exposed to PDF for 40 days. A significant fibrotic response can be observed with a larger ECM and many inflammatory cells. 

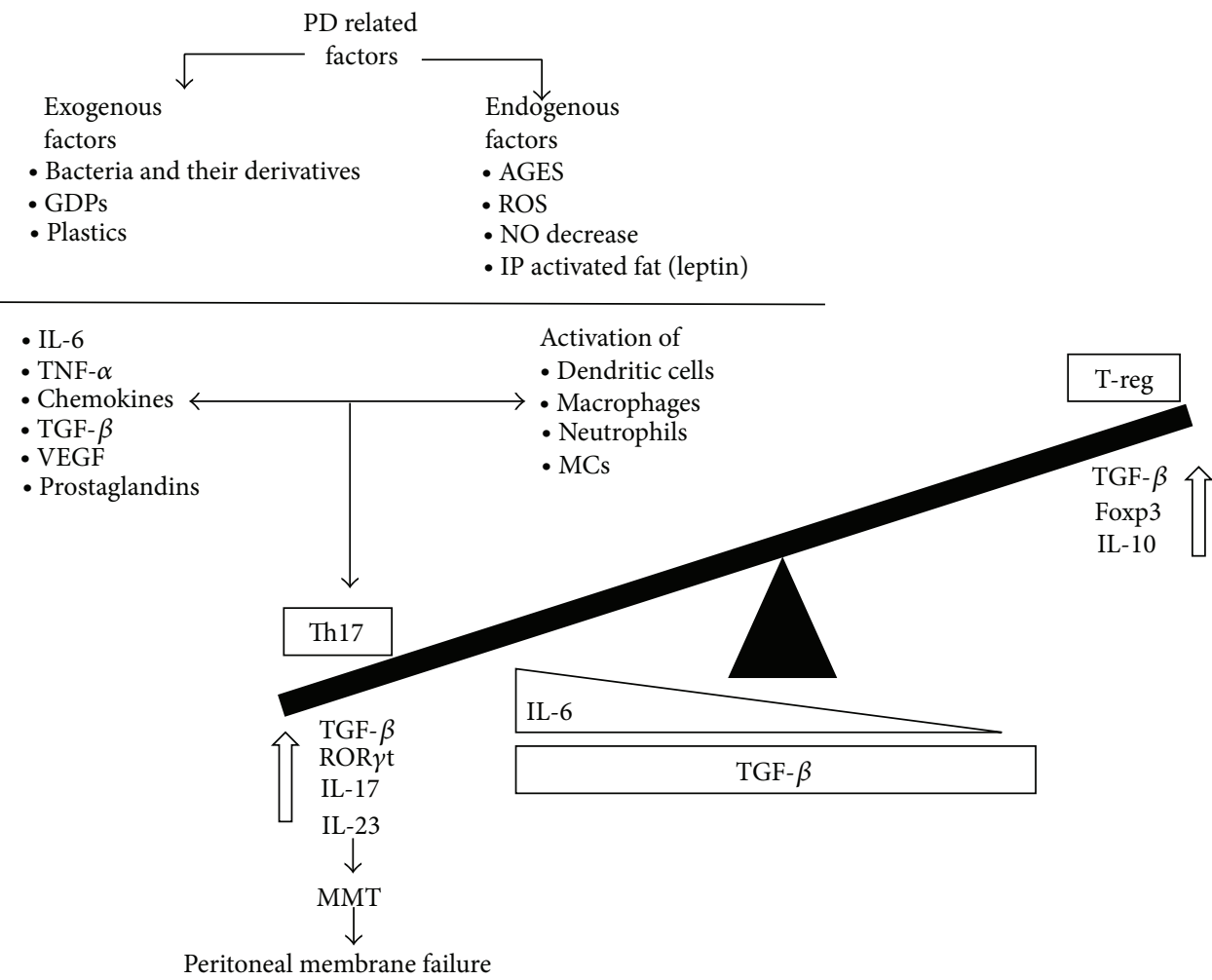

Figure 2: Mechanisms of Th17/Treg-balance in the peritoneal cavity in PD. Th17 predominance. In the peritoneal cavity local factors activate dendritic cells, macrophages, and neutrophils to produce proinflammatory molecules (IL-6, TNF $\alpha$, TGF- $\beta$, VEGF, chemokines, and prostaglandins). Immune cells and proinflammatory molecules are reciprocally activated; thus cells induce proinflammatory molecule release and vice versa. These molecules activate Th17 cells and IL-17 production, which may result in fibrosis and MMT. This occurs in the presence of continuously high TGF- $\beta$ levels. In contrast, in the presence of inactive immune cells together with low IL-6 levels, Treg cells are activated, releasing IL-10 and TGF- $\beta$ that may block MMT. Abbreviations: GDPs: glucose-derived products. IP: intraperitoneal. AGEs: advanced glycation end products.

highlighted the roles of Th17/Treg/Th1/Th2 responses in the pathogenesis of tissue fibrosis [46, 47]. Among these responses, Th17 cells may mediate strong inflammation by producing a cocktail of cytokines such as IL-6, IL-17A, IL-17F, and IL-22, among which IL-17A has been characterized as the major effector cytokine in causing a sustained inflammatory response.

Recent studies have investigated the role of Th17 response in fibrosis. It has been reported that administration of IL-17A in vitro increased the synthesis and secretion of collagen in alveolar epithelial cells in a pulmonary fibrosis model. Moreover, all IL-17-associated signaling pathways were mainly activated in fibrotic lung biopsies, and a blockade of IL-17A attenuated tissue injury, inflammation, and fibrosis in acute and chronic injuries [48].

Furthermore, IL-17 has also been reported to be involved in the pathogenesis of chronic liver fibrosis [44, 49-51]. The same mechanism was proposed in another study on liver damage in chronic hepatitis B patients who presented an elevated Th17 cells population [52].

Th17 cells also play a crucial role in autoimmune myocarditis, as based on in vitro and in vivo experiments which confirm that IL-17 induced cardiac fibrosis by activating the protein kinase $\mathrm{C}-\beta / \mathrm{Erk} 1 / 2 / \mathrm{NF}-\kappa \mathrm{B}$ pathway [53].
Moreover, the regulatory molecule CD69, through the regulation of Th17 effector responses, limits myocardial inflammation, fibrosis, and subsequent heart failure [54].

Recent studies have shown the importance of Th17 cells, and the hallmark cytokine IL-17A, in immune-mediated glomerulonephritis, including experimental antimyeloperoxidase glomerulonephritis, crescentic glomerulonephritis, and lupus nephritis $[55,56]$. Th17 cells participate in renal damage, as demonstrated by an experimental study in mice showing that Th17 cell injection caused albuminuria and neutrophils infiltration in the kidney [57]. Recent studies also show the presence of Th17 cells and elevated renal production of IL-17A in nonimmune experimental renal diseases, including a model of unilateral ureteral obstruction [58]. In experimental ischaemia reperfusion, neutrophils, but not Th17 cells, were the main sources of IL-17A and contribute to renal injury by natural killer T activation and IL-12/IFN- $\gamma$ production [59]. In renal allograft rejection, positive staining for IL-17A has been detected in tubular cells [60], as we have observed in an experimental model of CCN2-mediated renal damage, suggesting that renal cells could produce this cytokine and contribute to extending the damage. With this model we recently demonstrated that a blockade of IL-17A diminished renal inflammation [58]. 
Based on all the above studies, IL-17 has been proposed as a drug target in many fibrotic diseases [61].

\section{Regulatory T Cells and Tissue Fibrosis}

Although an induced Th17 response is connected to fibrogenesis, a relative decrease in the number of Treg cells may also be involved in the pathogenesis of inflammatory and fibrotic diseases. The evaluation of these cells in the context of experimentally induced fibrosis has been challenging, and Treg depletion in mice has been demonstrated to attenuate the development of lung fibrosis [62]. Moreover, in an experimental animal model of cardiac fibrosis, the depletion of Treg cells and/or adoptive transfer of isolated Tregs ameliorated cardiac fibrosis, indicating a protective role of regulatory $\mathrm{T}$ cell in tissue fibrosis [63]. Recently, it has been reported that Treg cells are essential for preventing from accumulation of fibrocytes and collagen deposition in a pulmonary disease animal model. In this study, it was shown that a blockade of Treg cells increased the accumulation of solid collagen and progression of the disease [64]. Finally, in another study (Keloid fibrotic disease) the potential role of Treg cells in attenuating collagen synthesis was investigated. This group found that the imbalance of Tregs may contribute to the development of this fibrotic disease and that the correction of this imbalance may be of therapeutic value [65].

\section{Th17 and Treg Lymphocyte Subsets in the Peritoneal Cavity on PD}

PD-related factors locally stimulate Th17 cells and can be subdivided into two groups: exogenous and endogenous. The exogenous factors include bacteria and their derivatives, which enter into the peritoneal cavity through PD-catheter or via intestinal translocation and can provoke peritonitis episodes [66]. Peritoneal endogenous factors such as AGEs [67] could be involved in the induction of IL-17 levels by activating IL- 6 and TGF- $\beta$ proinflammatory cytokines, respectively. Although there is little data regarding how advanced glycation end products (AGEs) are implicated in peritoneal dialysis damage, their implication in posttransplantation and diabetic kidneys showed an induction of IL6 and TGF- $\beta$, which are promoters of Th17 differentiation (Figure 2). Thus, it is plausible that the induced Th17 activity may have a poor fate in peritoneal damage during peritoneal dialysis. In nondiabetic PD patients, an elevation of IL17 in peritoneal cavity effluents followed by a peritonitis episode was demonstrated [66], which is one of the main complications that lead to peritoneal fibrosis in PD patients [3].

Currently, it is accepted that mesothelial to mesenchymal transition (MMT) is a key process in PM survival in PD. Mesothelial cells lose their basolateral and basoapical polarity, acquiring a fibroblastoid phenotype with migration capacity. The cells invade the submesothelial compact zone where they synthesize ECM and VEGF, which are responsible for fibrosis and angiogenesis, respectively [68, 69]. IL-17 itself is capable of inducing epithelial to mesenchymal transition in bronchial cells [70]. Although this effect has not been demonstrated in the peritoneum, it is very likely that IL-17 also contributes to PM deterioration via MMT induction.

The definitive evidence on IL-17 involvement in PM damage on PD was recently provided by Rodrigues-Díez et al. (2014). This study demonstrated in both mice and human samples that IL-17 is overexpressed in peritoneal biopsies. This was the first report to demonstrate that IL-17 participates in the typical fibrotic changes suffered in PM during longterm PD (induced fibronectin, $\alpha$-smooth muscle actin, and fibroblast specific protein-1 expression). Moreover, to better elucidate the effects of IL-17 on PM, intraperitoneal IL-17 was injected in mice, reproducing the changes that normally take place in PD patients. On the other hand, the use of a neutralizing IL-17A antibody injected intraperitoneally in mice exposed to PDF for 35 days blockaded the anatomical changes in the PM and reduced peritoneal fibrosis [71].

On the other hand, Treg cells are strongly connected with immune tolerance. Patients in end-stage renal disease with their suppressed immune response suffer impaired Treg cell responses [72]. Currently, there is not much evidence regarding the role of Treg cells in the peritoneal cavity during PD. To our knowledge, there is one study focusing on the function of Treg cells on peritoneal damage. This study concluded that rosiglitazone, a PPAR $\gamma$ agonist, augments the intraperitoneal IL-10 levels (Treg-associated cytokine), increases the recruitment of $\mathrm{CD}^{+} \mathrm{CD}^{+} 5^{+} \mathrm{FoxP}^{+}$(regulatory $\mathrm{T}$ cells), and finally attenuates peritoneal fibrosis in an experimental mouse PD model [73].

Evidence suggests that Th17 cells share common progenitors with Treg cells and that the developmental pathways of these two subsets are reciprocally regulated [74]. In fact, it has been recently demonstrated that a reduction in IL17 secretion due to Treg cell activation is associated with a diminished fibrotic response specifically in PD. The vitamin $\mathrm{D}$ pathway has been shown to regulate inflammatory responses. In regard to this, the effect of paricalcitol, a vitamin $\mathrm{D}$ receptor activator, was tested in a mouse model of PD to evaluate its effect on inflammatory cells and on the outcome of peritoneal fibrosis. It was found that the group that was treated with PDF presented increased levels of IL17 cytokine in the peritoneal effluents compared with the group that was treated with paricalcitol diluted in the PDF. Moreover, the increased IL-17 concentration was perfectly correlated with the thickness of the peritoneum, meaning IL-17 is a profibrotic cytokine. This effect was related to an increased number of Tregs in the group that was treated with paricalcitol [75].

\section{Therapeutic Approaches to Prevent Peritoneal Damage Using the Th17/Treg-Axis: From Animal Models to PD Patients}

Accepting the evidence above that Th17 and Treg subsets are involved in peritoneal damage in $\mathrm{PD}$, we propose the following therapeutic strategies. 


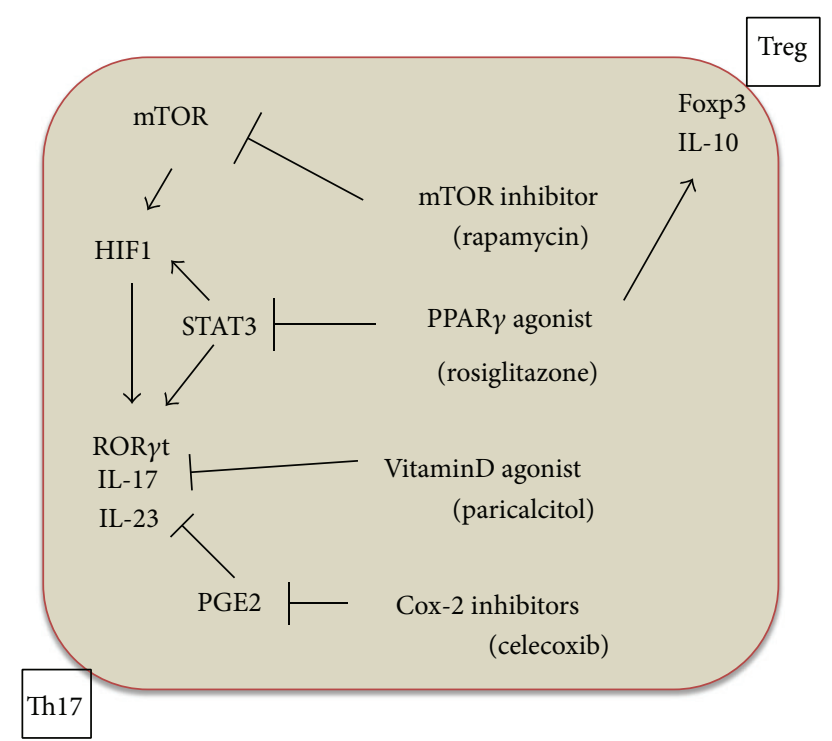

FIgURE 3: Therapeutic approaches to prevent peritoneal damage using the Th17/Treg-axis as a target. One of the most important modulators of Th17/Treg activity is the concentration of branch chain amino acids (BCAA). Although the data in relation to BCAA plasma levels in a uraemic state are contradictory, many articles indicate that these amino acids are decreased in uraemia due to systemic acidosis, inflammation, amino acid misbalances, and liquid overload. Normal or relative normal BCAA levels activate an mTOR (mammalian Target of Rapamycin) cascade including HIF1 and ROR $\gamma \mathrm{t}$ and subsequently IL-17 and IL-23 production. Rapamycin, an mTOR inhibitor, would block this cascade, thus providing an anti-inflammatory/antifibrotic and possibly anti-MMT effect. Moreover, PPAR $\gamma$ agonists can also inhibit Thl7 differentiation through a direct blockade of Stat3 transcription factor and HIF-1. Ultimately ROR $\gamma$ t is downregulated, and IL-17 and IL-23 production is decreased. However the PPRA $\gamma$ agonists are also able to act on the anti-inflammatory cascade. In the peritoneal cavity in $\mathrm{PD}$, rosiglitazone augments IL-10 levels and Treg activity (upregulation of $\mathrm{FoxP}^{+}$). This process could be one of the most important mechanisms by which PPRA $\gamma$ agonists protect the PM. In addition, paricalcitol, a specific vitamin D activator, has been recently shown to inhibit IL-17 production and PM fibrosis and possibly decrease MMT. Finally, celecoxib, a Cox2 inhibitor, decreases IL-17 production by blocking the E2 prostaglandin levels and thus attenuates the PM damage induced by PDF.

\subsection{Use Drugs and Molecular Strategies}

6.1.1. mTOR Inhibitors. The mTOR inhibition by Rapamycin may diminish IL-17 production. The mTOR activation induces hypoxia induced factor-1 (HIF-1) and ROR $\gamma \mathrm{t}$ activation and subsequently IL-17 and IL-23 production [76]. Thus, these drugs may provide an anti-inflammatory/antifibrotic effect and possibly an anti-MMT action as was demonstrated by Aguilera et al. [77] (Figure 3).

6.1.2. Peroxisome Proliferator-Activated Receptors- (PPAR-) $\gamma$ Agonists. The use of PPAR $\gamma$ agonists, for example, rosiglitazone, may be a therapeutic alternative to prevent peritoneal damage [78]. These receptors show a double protective effect.
First, they inhibit the Th17 differentiation via a Stat 3 cascade blockade, which results in a downregulation of ROR $\gamma \mathrm{t}$ and a decrease in IL-17 production [79]. Moreover, in an animal model study in which the effect of rosiglitazone in the preservation of the peritoneal membrane was investigated, it was found that rosiglitazone augmented the intraperitoneal IL-10 levels (Treg-associated cytokine) and increased the recruitment of $\mathrm{CD}^{+} \mathrm{CD} 25^{+} \mathrm{FoxP}^{+}$(regulatory T cells) [73] (Figure 3).

6.1.3. Vitamin D Receptor Activators. Another interesting drug that could ultimately have a beneficial effect on PM in PD is paricalcitol. Using a mice PD model, it was demonstrated that the PM thickening was reduced in mice treated with paricalcitol in comparison with the nontreated group. Moreover, in the effluents of mice treated with paricalcitol, an increased number of Treg cells and lower IL-17 levels were found in comparison with the nontreated group. In addition, the IL-17 levels measured in peritoneal effluent showed a positive linear correlation with the PM thickness [75]. These data suggest a direct involvement of IL-17 in peritoneal injury and paricalcitol may act on this target (Figure 3).

6.1.4. COX-2 Inhibitors. Celecoxib, a cyclooxygenase- (Cox-) 2 inhibitor agent, was shown to prevent the PM damage in $\mathrm{PD}$ when administrated orally to a group of mice in PD, acting directly on the inflammatory cascade in general. After 5 weeks of treatment, the celecoxib group showed a fibrotic response similar to healthy controls, while the untreated group exposed to PD only developed considerable fibrosis [19] (Figure 3).

\section{Discussion}

One of the most devastating complications of PD treatment is peritoneal fibrosis. The endogenous and exogenous factors mentioned above related to PDF generate a chronic inflammatory response in the peritoneal cavity. The balance between Th17 and Treg cells is guided by proinflammatory cytokines secreted from Th17 cells and anti-inflammatory cytokines produced from regulatory T cells. Any factor that may alter this balance can lead to peritoneal deterioration and finally to peritoneal damage. The high levels of various proinflammatory molecules such as IL- 6 and TGF- $\beta$ cytokines during PD create an environment that induces a chronic inflammatory condition in the peritoneal cavity and generates peritoneal fibrosis. This emergent concept suggests that the immune imbalance is the fundamental key for PM deterioration in PD.

Moreover, Th17 signaling with high IL-17 levels has been implicated in the aetiology of several types of inflammatory and fibrotic diseases. Therefore, components of the IL-17 and Treg cells pathway are considered highly "druggable" and are important targets for the treatment of these inflammatory and fibrotic diseases. Current evidence indicates that IL17 inhibition and Treg activation are logical therapeutic strategies for the treatment of animal peritoneal fibrosis $[71,73,75]$. 
In conclusion, the importance of developing new therapies to protect the peritoneal membrane blocking the IL-17 secretion or activating the Treg pathway has been demonstrated. Some novel therapeutic strategies tested in animal models and in vitro include the administration of $\mathrm{m}$-Tor inhibitors, PPAR $\gamma$ agonists, vitamin $\mathrm{D}$ receptor activators, and Cox-2 inhibitors. Lessons learned from regulators of Th17/Treg-axis may aid in the future clinical implementation of these agents with the goal of reducing peritoneal fibrosis and improving patients' life undergoing PD.

\section{Conclusion}

PD-related factors are responsible for Th17 activation/Treg deactivation in the peritoneal cavity during peritoneal dialysis. The Th17/Treg-axis is important for maintaining the anatomical and functional integrity of the PM. Therefore, the Th17/Treg-axis may be considered a future therapeutic target.

\section{Conflict of Interests}

The authors declare that there is no conflict of interests regarding the publication of this paper.

\section{Acknowledgments}

This work was supported in part by grants from Ministerio de Economia y competitividad SAF2010-21249 to Manuel López-Cabrera, Comunidad Autónoma de Madrid 2010BMD2321 (FIBROTEAM) to Manuel Lopez Cabrera, and Fondo de Investigaciones Santitarias RETICS 06/0016 and PI 09/0064 to Rafael Selgas and FIS 12/01175 to Abelardo Aguilera Peralta. Georgios Liappas is fully supported from European Union, Seventh Framework Program "EuTRiPD," under Grant Agreement PITN-GA-2011-287813. The authors would like to thank Juliette Siegfried and her team at ServingMed.com for editing the language of the paper.

\section{References}

[1] J. D. Williams, K. J. Craig, N. Topley et al., "Morphologic changes in the peritoneal membrane of patients with renal disease," Journal of the American Society of Nephrology, vol. 13, no. 2, pp. 470-479, 2002.

[2] C. Chaimovitz, "Peritoneal dialysis," Kidney International, vol. 45, no. 4, pp. 1226-1240, 1994.

[3] R. T. Krediet, "The peritoneal membrane in chronic peritoneal dialysis," Kidney International, vol. 55, no. 1, pp. 341-356, 1999.

[4] J. Waniewski, "Peritoneal fluid transport: mechanisms, pathways, methods of assessment," Archives of Medical Research, vol. 44, no. 8, pp. 576-583, 2013.

[5] J. Nevado, C. Peiró, S. Vallejo et al., "Amadori adducts activate nuclear factor-kappaB-related proinflammatory genes in cultured human peritoneal mesothelial cells," British Journal of Pharmacology, vol. 146, no. 2, pp. 268-279, 2005.

[6] G. R. Silberhumer, H. Pokorny, G. Györi, and F. Mühlbacher, "Surgical aspects of peritoneal dialysis," Wiener Medizinische Wochenschrift, vol. 163, no. 11-12, pp. 288-294, 2013.

[7] L. F. Fried, J. Bernardini, J. R. Johnston, and B. Piraino, "Peritonitis influences mortality in peritoneal dialysis patients,"
Journal of the American Society of Nephrology, vol. 7, no. 10, pp. 2176-2182, 1996.

[8] M. A. M. Mateijsen, A. C. van der Wal, P. M. E. M. Hendriks et al., "Vascular and interstitial changes in the peritoneum of CAPD patients with peritoneal sclerosis," Peritoneal Dialysis International, vol. 19, no. 6, pp. 517-525, 1999.

[9] L. S. Aroeira, A. Aguilera, J. A. Sánchez-Tomero et al., "Epithelial to mesenchymal transition and peritoneal membrane failure in peritoneal dialysis patients: Pathologic significance and potential therapeutic interventions," Journal of the American Society of Nephrology, vol. 18, no. 7, pp. 2004-2013, 2007.

[10] A. Lupo, C. Rugiu, A. Lapolla et al., "The dialytic failure of the peritoneal membrane," Contributions to Nephrology, vol. 131, pp. 90-96, 2001.

[11] J. Plum, S. Hermann, A. Fusshöller et al., "Peritoneal sclerosis in peritoneal dialysis patients related to dialysis settings and peritoneal transport properties," Kidney International, Supplement, vol. 59, no. 78, pp. S42-S47, 2001.

[12] O. Devuyst, P. J. Margetts, and N. Topley, "The pathophysiology of the peritoneal membrane," Journal of the American Society of Nephrology, vol. 21, no. 7, pp. 1077-1085, 2010.

[13] K. Wieczorowska-Tobis, A. Styszynski, A. Breborowicz, and D. G. Oreopoulos, "Comparison of the biocompatibility of phosphate-buffered saline alone, phosphate-buffered saline supplemented with glucose, and dianeal 3.86\%," Peritoneal Dialysis International, vol. 21, no. 3, pp. S362-S364, 2001.

[14] C. J. Holmes, "Biocompatibility of peritoneal dialysis solutions," Peritoneal Dialysis International, vol. 13, no. 2, pp. 88-94, 1993.

[15] A. J. Collins, W. Hao, H. Xia et al., "Mortality risks of peritoneal dialysis and hemodialysis," The American Journal of Kidney Diseases, vol. 34, no. 6, pp. 1065-1074, 1999.

[16] R. Selgas, M.-J. Fernandez-Reyes, E. Bosque et al., "Functional longevity of the human peritoneum: how long is continuous peritoneal dialysis possible? Results of a prospective medium long-term study," American Journal of Kidney Diseases, vol. 23, no. 1, pp. 64-73, 1994.

[17] R. Pecoits-Filho, P. Stenvinkel, A. Yee-Moon Wang, O. Heimbürger, and B. Lindholm, "Chronic inflammation in peritoneal dialysis: the search for the holy grail?" Peritoneal Dialysis International, vol. 24, no. 4, pp. 327-339, 2004.

[18] G. T. González-Mateo, J. Loureiro, J. A. Jiménez-Hefferman et al., "Chronic exposure of mouse peritoneum to peritoneal dialysis fluid: structural and functional alterations of the peritoneal membrane," Peritoneal Dialysis International, vol. 29, no. 2, pp. 227-230, 2009.

[19] L. S. Aroeira, E. Lara-Pezzi, J. Loureiro et al., "Cyclooxygenase-2 mediates dialysate-induced alterations of the peritoneal membrane," Journal of the American Society of Nephrology, vol. 20, no. 3, pp. 582-592, 2009.

[20] I. Griveas, G. Visvardis, A. Fleva et al., "Lymphocytes subsets in the course of continuous ambulatory peritoneal dialysis (CAPD)," Renal Failure, vol. 26, no. 6, pp. 641-646, 2004.

[21] A. Glik and A. Douvdevani, "T lymphocytes: the 'cellular' arm of acquired immunity in the peritoneum," Peritoneal Dialysis International, vol. 26, no. 4, pp. 438-448, 2006.

[22] T. R. Mosmann and R. L. Coffman, "TH1 and TH2 cells: different patterns of lymphokine secretion lead to different functional properties," Annual Review of Immunology, vol. 7, pp. 145-173, 1989.

[23] T. Yokoyama, K. Nitta, K. Futatsuyama et al., "Identification of T helper cell subsets in continuous ambulatory peritoneal dialysis patients," Nephron, vol. 89, no. 2, pp. 215-218, 2001. 
[24] A. Brauner, B. Hylander, and B. Wretlind, "Tumor necrosis factor- $\alpha$, interleukin- $1 \beta$, and interleukin-1 receptor antagonist in dialysate and serum from patients on continuous ambulatory peritoneal dialysis," The American Journal of Kidney Diseases, vol. 27, no. 3, pp. 402-408, 1996.

[25] H.-H. Wang and C.-Y. Lin, "Interleukin-12 and -18 levels in peritoneal dialysate effluent correlate with the outcome of peritonitis in patients undergoing peritoneal dialysis: implications for the type I/type II T-cell immune response," American Journal of Kidney Diseases, vol. 46, no. 2, pp. 328-338, 2005.

[26] P. Martín, M. Gómez, A. Lamana et al., "CD69 association with Jak3/Stat5 proteins regulates Th17 cell differentiation," Molecular and Cellular Biology, vol. 30, no. 20, pp. 4877-4889, 2010.

[27] E. Bettelli, Y. Carrier, W. Gao et al., "Reciprocal developmental pathways for the generation of pathogenic effector TH17 and regulatory T cells," Nature, vol. 441, no. 7090, pp. 235-238, 2006.

[28] D. R. Littman and A. Y. Rudensky, "Th17 and regulatory T cells in mediating and restraining inflammation," Cell, vol. 140, no. 6, pp. 845-858, 2010.

[29] J. C. Waite and D. Skokos, "Th17 response and inflammatory autoimmune diseases," International Journal of Inflammation, vol. 2012, Article ID 819467, 10 pages, 2012.

[30] I. I. Ivanov, B. S. McKenzie, L. Zhou et al., "The orphan nuclear receptor ROR $\gamma$ t directs the differentiation program of proinflammatory IL-17 ${ }^{+}$T helper cells," Cell, vol. 126, no. 6, pp. 1121-1133, 2006.

[31] T. J. Harris, J. F. Grosso, H.-R. Yen et al., "An in vivo requirement for STAT3 signaling in TH17 development and TH17-dependent autoimmunity," Journal of Immunology, vol. 179, no. 7, pp. 43134317, 2007.

[32] X. O. Yang, A. D. Panopoulos, R. Nurieva et al., "STAT3 regulates cytokine-mediated generation of inflammatory helper T cells," Journal of Biological Chemistry, vol. 282, no. 13, pp. 9358-9363, 2007.

[33] L. Zhou, J. E. Lopes, M. M. W. Chong et al., “TGF-B-induced Foxp3 inhibits $\mathrm{T}_{H} 17$ cell differentiation by antagonizing ROR $\gamma \mathrm{t}$ function," Nature, vol. 453, no. 7192, pp. 236-240, 2008.

[34] J. M. Kim, J. P. Rasmussen, and A. Y. Rudensky, "Regulatory $\mathrm{T}$ cells prevent catastrophic autoimmunity throughout the lifespan of mice," Nature Immunology, vol. 8, no. 2, pp. 191-197, 2007.

[35] K. Lahl, C. Loddenkemper, C. Drouin et al., "Selective depletion of Foxp $3^{+}$regulatory T cells induces a scurfy-like disease," Journal of Experimental Medicine, vol. 204, no. 1, pp. 57-63, 2007.

[36] M. Veldhoen, R. J. Hocking, C. J. Atkins, R. M. Locksley, and B. Stockinger, "TGF $\beta$ in the context of an inflammatory cytokine milieu supports de novo differentiation of IL-17-producing T cells," Immunity, vol. 24, no. 2, pp. 179-189, 2006.

[37] P. R. Mangan, L. E. Harrington, D. B. O'Quinn et al., “Transforming growth factor- $\beta$ induces development of the $\mathrm{T}_{H} 17$ lineage," Nature, vol. 441, no. 7090, pp. 231-234, 2006.

[38] H. P. Eugster, K. Frei, M. Kopf, H. Lassmann, and A. Fontana, "IL-6-deficient mice resist myelin oligodendrocyte glycoprotein-induced autoimmune encephalomyelitis," European Journal of Immunology, vol. 28, no. 7, pp. 2178-2187, 1998.

[39] E. B. Samoilova, J. L. Horton, B. Hilliard, T.-S. T. Liu, and Y. Chen, "IL-6-deficient mice are resistant to experimental autoimmune encephalomyelitis: roles of IL-6 in the activation and differentiation of autoreactive T cells," Journal of Immunology, vol. 161, no. 12, pp. 6480-6486, 1998.
[40] T. Korn, A. C. Anderson, E. Bettelli, and M. Oukka, "The dynamics of effector T cells and Foxp $3^{+}$regulatory T cells in the promotion and regulation of autoimmune encephalomyelitis," Journal of Neuroimmunology, vol. 191, no. 1-2, pp. 51-60, 2007.

[41] S. Tamada, T. Asai, N. Kuwabara et al., "Molecular mechanisms and therapeutic strategies of chronic renal injury: the role of nuclear factor $\kappa \mathrm{B}$ activation in the development of renal fibrosis," Journal of Pharmacological Sciences, vol. 100, no. 1, pp. 17-21, 2006.

[42] L. M. Blanco-Colio, J. L. Martín-Ventura, B. Munoz-Garcia et al., "TWEAK and Fn14. New players in the pathogenesis of atherosclerosis," Frontiers in Bioscience, vol. 12, no. 10, pp. 36483655, 2007.

[43] T. A. Wynn, "Cellular and molecular mechanisms of fibrosis," The Journal of Pathology, vol. 214, no. 2, pp. 199-210, 2008.

[44] Z. Tan, X. Qian, R. Jiang et al., "IL-17A plays a critical role in the pathogenesis of liver fibrosis through hepatic stellate cell activation," Journal of Immunology, vol. 191, no. 4, pp. 1835-1844, 2013.

[45] S. A. Hasan, B. Eksteen, D. Reid et al., "Role of IL-17A and neutrophils in fibrosis in experimental hypersensitivity pneumonitis," The Journal of Allergy and Clinical Immunology, vol. 131, no. 6, pp. 1663.e5-1673.e5, 2013.

[46] H.-Z. Yang, B. Cui, H.-Z. Liu et al., "Targeting TLR2 attenuates pulmonary inflammation and fibrosis by reversion of suppressive immune microenvironment," The Journal of Immunology, vol. 182, no. 1, pp. 692-702, 2009.

[47] K. J. Aitken, C. Tolg, T. Panchal et al., "Mammalian target of rapamycin (mTOR) induces proliferation and dedifferentiation responses to three coordinate pathophysiologic stimuli (mechanical strain, hypoxia, and extracellular matrix remodeling) in rat bladder smooth muscle," The American Journal of Pathology, vol. 176, no. 1, pp. 304-319, 2010.

[48] S. Mi, Z. Li, H.-Z. Yang et al., "Blocking IL-17A promotes the resolution of pulmonary inflammation and fibrosis via TGFbetal-dependent and -independent mechanisms," Journal of Immunology, vol. 187, no. 6, pp. 3003-3014, 2011.

[49] L. Wang, S. Chen, and K. Xu, "IL-17 expression is correlated with hepatitis B-related liver diseases and fibrosis," International Journal of Molecular Medicine, vol. 27, no. 3, pp. 385-392, 2011.

[50] A. Lemmers, C. Moreno, T. Gustot et al., "The interleukin17 pathway is involved in human alcoholic liver disease," Hepatology, vol. 49, no. 2, pp. 646-657, 2009.

[51] D. Fenoglio, F. Bernuzzi, F. Battaglia et al., "Th17 and regulatory T lymphocytes in primary biliary cirrhosis and systemic sclerosis as models of autoimmune fibrotic diseases," Autoimmunity Reviews, vol. 12, no. 2, pp. 300-304, 2012.

[52] J.-Y. Zhang, Z. Zhang, F. Lin et al., "Interleukin-17-producing $\mathrm{CD} 4{ }^{+} \mathrm{T}$ cells increase with severity of liver damage in patients with chronic hepatitis B," Hepatology, vol. 51, no. 1, pp. 81-91, 2010.

[53] Y. Liu, H. Zhu, Z. Su et al., "IL-17 contributes to cardiac fibrosis following experimental autoimmune myocarditis by a PKC $\beta /$ Erk1/2/NF- $\kappa \mathrm{B}$-dependent signaling pathway," International Immunology, vol. 24, no. 10, pp. 605-612, 2012.

[54] A. Cruz-Adalia, L. J. Jiménez-Borreguero, M. Ramírez-Huesca et al., "CD69 limits the severity of cardiomyopathy after autoimmune myocarditis," Circulation, vol. 122, no. 14, pp. 1396-1404, 2010.

[55] A. R. Kitching and S. R. Holdsworth, "The emergence of Th17 cells as effectors of renal injury," Journal of the American Society of Nephrology, vol. 22, no. 2, pp. 235-238, 2011. 
[56] J.-E. Turner, H.-J. Paust, O. M. Steinmetz, and U. Panzer, "The Th17 immune response in renal inflammation," Kidney International, vol. 77, no. 12, pp. 1070-1075, 2010.

[57] S. A. Summers, O. M. Steinmetz, M. Li et al., "Th1 and Th17 cells induce proliferative glomerulonephritis," Journal of the American Society of Nephrology, vol. 20, no. 12, pp. 2518-2524, 2009.

[58] R. Rodrigues-Díez, R. R. Rodrigues-Díez, S. Rayego-Mateos et al., "The C-terminal module IV of connective tissue growth factor is a novel immune modulator of the Th17 response," Laboratory Investigation, vol. 93, no. 7, pp. 812-824, 2013.

[59] L. Li, L. Huang, A. L. Vergis et al., "IL-17 produced by neutrophils regulates IFN- $\gamma$-mediated neutrophil migration in mouse kidney ischemia-reperfusion injury," The Journal of Clinical Investigation, vol. 120, no. 1, pp. 331-342, 2010.

[60] A. Loverre, T. Tataranni, G. Castellano et al., "IL-17 expression by tubular epithelial cells in renal transplant recipients with acute antibody-mediated rejection," The American Journal of Transplantation, vol. 11, no. 6, pp. 1248-1259, 2011.

[61] S. Ivanov and A. Lindén, "Interleukin-17 as a drug target in human disease," Trends in Pharmacological Sciences, vol. 30, no. 2, pp. 95-103, 2009.

[62] F. Liu, J. Liu, D. Weng et al., "CD4+CD25+Foxp3+ regulatory T cells depletion may attenuate the development of silica-induced lung fibrosis in mice," PLoS ONE, vol. 5, no. 11, Article ID e15404, 2010.

[63] Y. Cao, W. Xu, and S. Xiong, "Adoptive transfer of regulatory $\mathrm{T}$ Cells protects against Coxsackievirus B3-induced cardiac fibrosis," PLoS ONE, vol. 8, no. 9, Article ID e74955, 2013.

[64] X. Peng, M. W. Moore, H. Peng et al., "CD4+CD25+FoxP3+ regulatory tregs inhibit fibrocyte recruitment and fibrosis via suppression of FGF-9 production in the TGF- $\beta 1$ exposed murine lung," Frontiers in Pharmacology, vol. 5, article 80, 2014.

[65] N. Murao, K.-I. Seino, T. Hayashi et al., "Treg-enriched CD4+ T cells attenuate collagen synthesis in keloid fibroblasts," Experimental Dermatology, vol. 23, no. 4, pp. 266-271, 2014.

[66] H.-H. Wang, T.-Y. Lee, and C.-Y. Lin, "Kinetics and involvement of interleukin-17 in the outcome of peritonitis in nondiabetic patients undergoing peritoneal dialysis," Journal of the Chinese Medical Association, vol. 74, no. 1, pp. 11-15, 2011.

[67] D. Maksić, M. Colić, V. Stanković-Popović, M. Radojević, and D. Bokonjić, "Systemic and intraperitoneal proinflammatory cytokines profiles in patients on chronic peritoneal dialysis," Medicinski Pregled, vol. 60, supplement 2, pp. 53-57, 2007.

[68] J. Loureiro, G. Gónzalez-Mateo, J. Jimenez-Heffernan, R. Selgas, M. López-Cabrera, and A. Aguilera Peralta, "Are the mesothelial-to-mesenchymal transition, sclerotic peritonitis syndromes, and encapsulating peritoneal sclerosis part of the same process?" International Journal of Nephrology, vol. 2013, Article ID 263285, 7 pages, 2013.

[69] M. Yáñez-Mó, E. Lara-Pezzi, R. Selgas et al., "Peritoneal dialysis and epithelial-to-mesenchymal transition of mesothelial cells," The New England Journal of Medicine, vol. 348, no. 5, pp. 403413, 2003.

[70] X. Ji, J. Li, L. Xu et al., "IL4 and IL-17A provide a Th2/Th17polarized inflammatory milieu in favor of TGF- $\beta 1$ to induce bronchial epithelial-mesenchymal transition (EMT)," International Journal of Clinical and Experimental Pathology, vol. 6, no. 8, pp. 1481-1492, 2013.

[71] R. Rodrigues-Díez, L. S. Aroeira, M. Orejudo et al., "IL-17A is a novel player in dialysis-induced peritoneal damage," Kidney International, vol. 86, pp. 303-315, 2014.
[72] T. K. Hendrikx, E. A. F. J. Van Gurp, W. M. Mol et al., "End-stage renal failure and regulatory activities of CD4 +CD25bright+FoxP3+ T-cells," Nephrology Dialysis Transplantation, vol. 24, no. 6, pp. 1969-1978, 2009.

[73] P. Sandoval, J. Loureiro, G. González-Mateo et al., "PPAR$\gamma$ agonist rosiglitazone protects peritoneal membrane from dialysis fluid-induced damage," Laboratory Investigation, vol. 90, no. 10, pp. 1517-1532, 2010.

[74] A. Ueda, L. Zhou, and P. L. Stein, "Fyn promotes Th17 differentiation by regulating the kinetics of ROR $\gamma \mathrm{t}$ and Foxp3 expression," Journal of Immunology, vol. 188, no. 11, pp. 52475256, 2012.

[75] G. T. González-Mateo, V. Fernández-Míllara, T. Bellón et al., "Paricalcitol reduces peritoneal fibrosis in mice through the activation of regulatory $\mathrm{T}$ cells and reduction in IL-17 production," PLoS ONE, vol. 9, no. 10, Article ID e108477, 2014.

[76] A. Ikejiri, S. Nagai, N. Goda et al., "Dynamic regulation of Th17 differentiation by oxygen concentrations," International Immunology, vol. 24, no. 3, pp. 137-146, 2012.

[77] A. Aguilera, L. S. Aroeira, M. Ramírez-Huesca et al., "Effects of rapamycin on the epithelial-to-mesenchymal transition of human peritoneal mesothelial cells," International Journal of Artificial Organs, vol. 28, no. 2, pp. 164-169, 2005.

[78] S. G. Kim and W. H. Lee, "AMPK-dependent metabolic regulation by PPAR agonists," PPAR Research, vol. 2010, Article ID 549101, 10 pages, 2010.

[79] L. Klotz, S. Burgdorf, I. Dani et al., "The nuclear receptor PPARgamma selectively inhibits Th17 differentiation in a T cell-intrinsic fashion and suppresses CNS autoimmunity," The Journal of Experimental Medicine, vol. 206, no. 10, pp. 20792089, 2009.

[80] A. Aguilera, J. Loureiro, G. Gónzalez-Mateo, R. Selgas, and M. López-Cabrera, "The mesothelial to mesenchymal transition a pathogenic and therapeutic key for peritoneal membrane failure," in The Latest in Peritoneal Dialysis, chapter 2, InTech, 2013. 


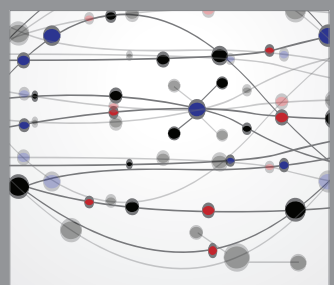

The Scientific World Journal
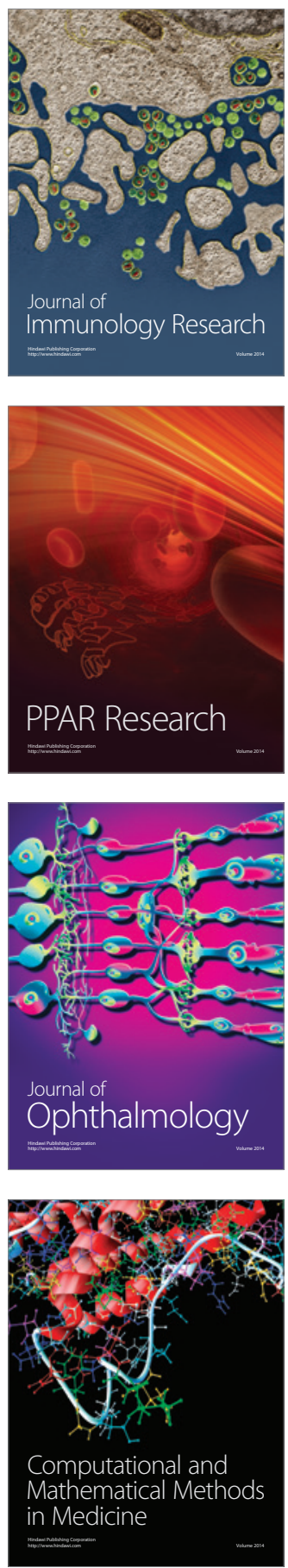

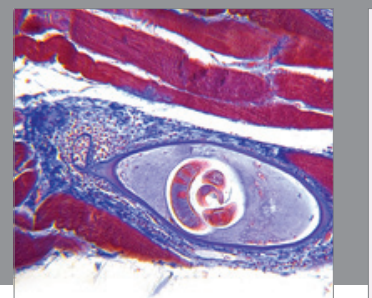

Gastroenterology

Research and Practice
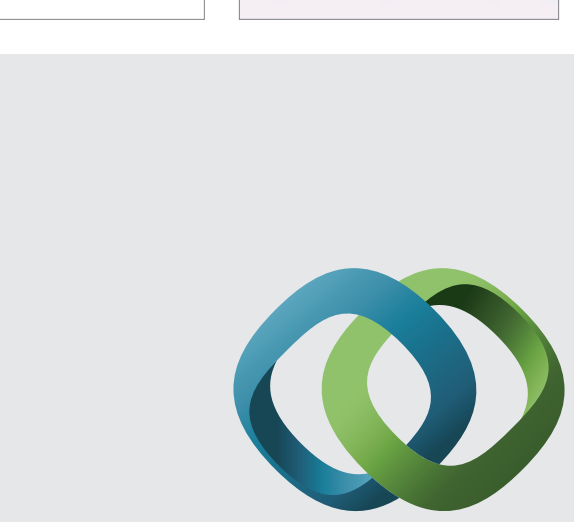

\section{Hindawi}

Submit your manuscripts at

http://www.hindawi.com
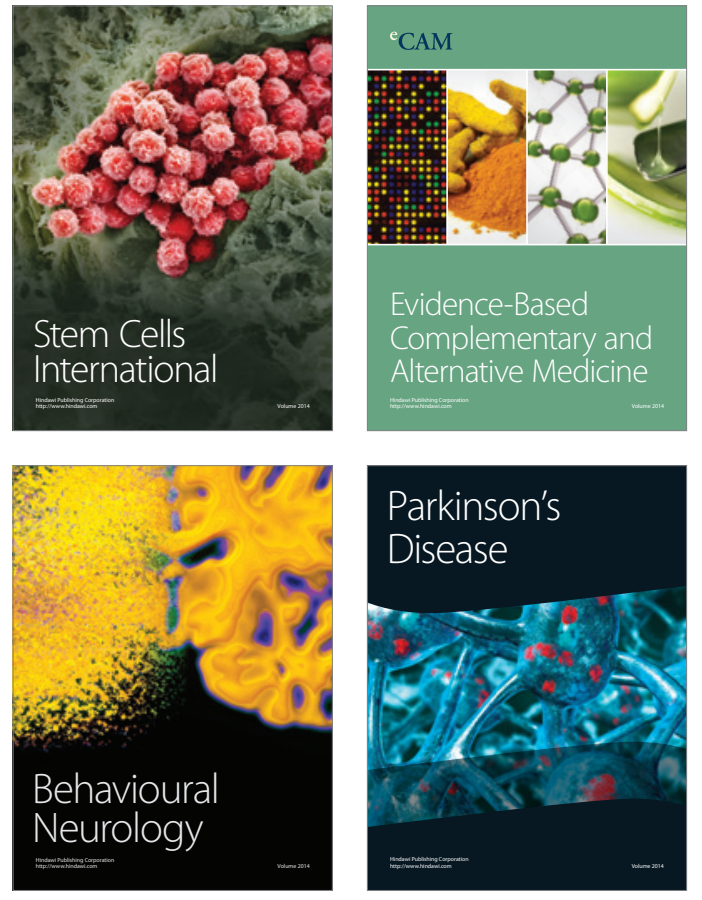
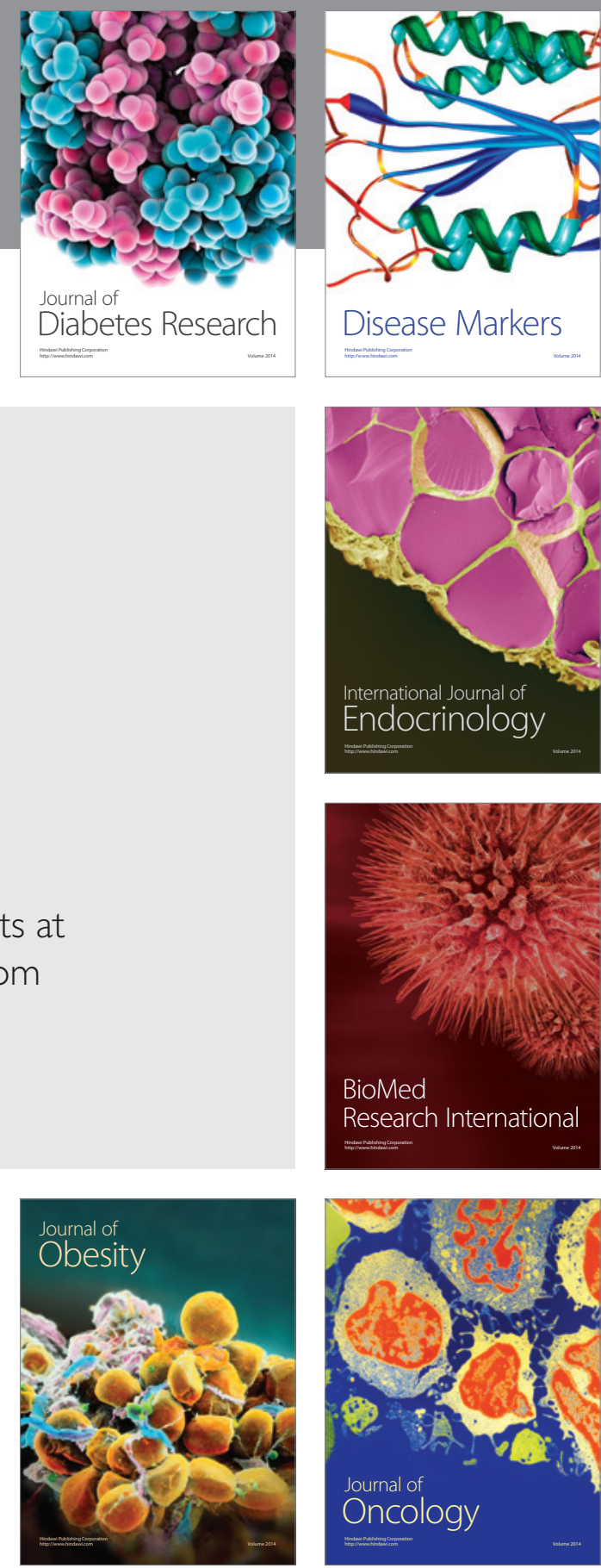

Disease Markers
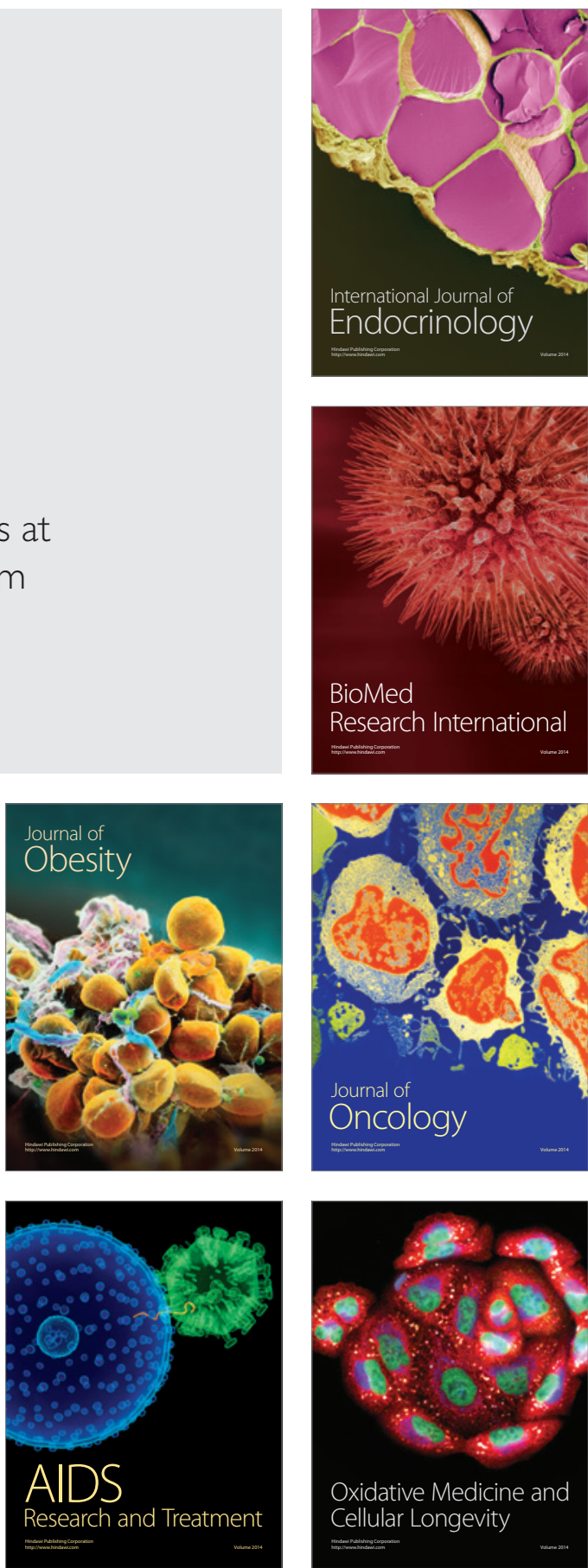